\title{
A Review of the Current Research on the Translation of Emperor Internal Chinese Medicine*
}

\author{
Juan Wang \\ School of Foreign Languages \\ Hubei University of Chinese Medicine \\ Wuhan, China 430065
}

\begin{abstract}
Among the four classics of traditional Chinese medicine, domestic scholars have made the most abundant achievements in the translation of Emperor Internal Chinese Medicine. The research mainly focuses on the four aspects of diachronic review and sorting out of the translation of the masterpiece, the commentary on the single translation or the comparative study of multiple versions, the attention paid to the translation methodology at the micro-linguistic level of the translation, and the tentative combination of various contemporary translation theories and the translation study of Emperor Internal Chinese Medicine. However, the existing studies pay too much attention to the text itself and the color of practice is still strong with slightly inadequate theoretical constructiveness. Besides that, the hypertext factors such as social and cultural context in the production of the translation are neglected.
\end{abstract}

Keywords-Emperor Internal Chinese Medicine; microscopic; theory construction; hypertext

\section{INTRODUCTION}

Featured by profound words and thoughts, Emperor Internal Chinese Medicine is recognized as the school of medical classics, the source of TCM theory. Among the four classics of traditional Chinese medicine, Emperor Internal Chinese Medicine enjoys the largest number of translated versions and the largest number of literatures on its translation and introduction, which is far superior to other counterparts. According to the incomplete statistics of CNKI, there are as many as seventy or eighty papers including doctoral dissertations. As far as the translators of Emperor Internal Chinese Medicine are concerned, most of them have the background of traditional Chinese medicine, or are teachers engaged in foreign language teaching of traditional Chinese medicine, such as Shi Yunzhong, Yao Xin, Jiang Jichang and other scholars active in the front line of TCM translation research, or graduate students in the direction of English of TCM. Some graduate students from other comprehensive universities or foreign language universities, such as Fudan University, Lanzhou University, Northwest University of Agriculture and Forestry Science and

*This paper is one of the achievements of the humanities and social sciences research project "Reconstruction of the Classics of Traditional Chinese Medicine: Translation, Introduction and Overseas Dissemination of Emperor Internal Chinese Medicine" (18Q092) of the Ministry of Education of Hubei Province in 2018.
Technology, Beijing Foreign Studies University, choose the rendering of Emperor Internal Chinese Medicine as the topic of their graduation thesis, which shows that the English translation of classics of traditional Chinese medicine is attracting more and more attention. Considering the publication level, although there are many core journals of foreign languages such as Chinese Translators Journal and Chinese Science and Technology Translators Journal, most of them are published in core medical journals such as Chinese Journal of Integrated Traditional and Western Medicine, Chinese Journal of Basic Medicine in Traditional Chinese Medicine, Lishizhen Medicine and Materia Medical Research, Chinese Journal of Traditional Chinese Medicine and Pharmacy or journals of TCM-oriented universities, such as Journal of Shanghai University of Traditional Chinese Medicine, Journal of Liaoning University of Traditional Chinese Medicine, Journal of Shanxi University of Traditional Chinese Medicine, etc. . For the research content there are four following categories by and large.

\section{DIACHRONIC RETROSPECT, CAREFUL COMBINATION AND MACROSCOPIC VIEW ON THE TRANSLATION OF EMPEROR INTERNAL CHINESE MEDICINE}

Some scholars have made a macroscopic outline of the translation and introduction of Emperor Internal Chinese Medicine and reviewed the production process of the major translations diachronically. For example, Liu $\mathrm{Na}$, Wang $\mathrm{Na}$, Zhang Tingting and Wang Zhimei [1] believe that Emperor Internal Chinese Medicine, as the earliest medical ancient book in China, has many translated versions. Through a brief review of the English versions, they discuss the translators' understanding and translation of this medical masterpiece in different periods and academic backgrounds, holding that the English translation of Emperor Internal Chinese Medicine has shown the characteristics of flourishing and contending among hundreds of schools of thought. As far as translators are concerned, they have experienced the transformation from western translators to Chinese-western translators and then to Chinese translators, and the translators' academic backgrounds also have unfolded the trend of diversification. Xie Shuting [2] briefly reviews the English versions of Emperor Internal Chinese Medicine and makes a simple statistical analysis of the researchers who translate the writing. Then, she holds that descriptive translatology and corpus will bring new ideas to the study of Emperor Internal 
Chinese Medicine translation. Zhang Lin, Wenjuan and Jiang Jichang [3] make the research on the translation of the title of the book. Firstly, they sorted out the different translations of the titles of the 12 versions. Then, based on the principle of faithfulness to the English translation of the titles of ancient books of Chinese medicine, starting from the source, they explained the essential meanings of the title of Huang Di, Nei and Jing respectively followed by coming to the conclusion that Yellow Emperor's Canon of Medicine conveyed the original meaning to the greatest extent. Shen Feiyu [4] makes a detailed study of the historical background of this ancient works, believing that only by correctly understanding its meaning, can the title of the book be translated well, and suggests that Huangdi Neijing Suwen be translated into General Questions and Answers from Emperor Internal Chinese Medicine. Moreover, Shen Feiyu raises the issue of translator's literacy in translating Emperor Internal Chinese Medicine and divides the history of its translation into three stages, namely, the marginal development stage from 1925 to 1950 , the gradual development stage from 1978 to 1997 and the rapid development stage from 2001 to today.

It has been nearly a hundred years since the first English translation was published in 1925. During this period, 11 other versions have appeared one after another. However, the attention paid to them is unequal, especially to Li Zhaoguo, Wiess and Wenshu.

\section{COMMENTS ON THE SINGLE TRANSLATION OF EMPEROR INTERNAL CHINESE MEDICINE AND A COMPARATIVE STUDY OF SEVERAL VERSIONS}

For the different translations, the most researchers focus their eyes on versions of $\mathrm{De}, \mathrm{Wu}$ father and son, and Maoshing Ni. Shi Yunzhong, Ma Jiming and Xu Zheng [5] introduced in detail the reasons, process, characteristics, social evaluation and shortcomings of the translation of Emperor Internal Chinese Medicine by Ms. Ilza Veith. With the help of the academician of the Royal Australian Academy of Sciences, the Atlanta Medical Research Center in Australia and scholars from the Institute of History of Science and Medical Technology of Johns Hopkins University in the United States, they have obtained many valuable first-hand materials and become the first scholars to disclose the relevant information of the translation in detail. This paper points out that Veith's translation principle of "translating the contents of the Inner Classic only, without going deep into the meaning of the universe" and Ms. Ilza Veith's "notes" and "footnotes" which are often used to deal with the difficult contents are of great reference value to the English translation of this classic masterpiece.

In the process of translating Emperor Internal Chinese Medicine, Li Zhaoguo once published a text to reveal his mental process, explaining the translation principles and methods he used in his translation. He put forward the translation principle of "translating ancient texts without decoration". In 2009, Li Zhaoguo [6] published an article in Chinese Science and Technology Translators Journal, which further generalized the principles of translating Emperor Internal Chinese Medicine into English as follows: "integrating the medicine into philosophy to make the translation unity and coherence. To conform to the internal meaning, it's necessary to start from the externality because it embraces the profound ideas and subtle meaning. When making translation, the translator should stand in the perspective of ancients. Because of the refinement of conceptual metaphysics and the annotation of transliteration, transliteration and annotation shall be utilized. The translation of ancient text should follow the ancient style and the text should not be embellished for retaining its original truth. It's a powerful ability to reach an effect of being ambiguous. The same text may be translated in different ways to be in line with the original meaning. The derivative language should follow its ancient meaning correspondingly and when rendering, it's essential to hold a dialectical attitude." These articles are excellent materials for studying Li Zhaoguo's translation. Wang Xinyue [7] believes that Professor Li Zhaoguo's translation of Emperor Internal Chinese Medicine uses transliteration, literal translation, free translation, annotation and other techniques to minimize the errors of the essence of traditional Chinese medicine in external dissemination and communication. In his version, the criterion of "faithfulness, expressiveness and elegance" are described in all aspects. Huang $\mathrm{Yu}$ [8] applied the theory of eco-translatology to study the English translation of $\mathrm{Li}$ Zhaoguo's General Questions and Answers from Emperor Internal Chinese Medicine, and analyzed the translation through descriptive and case studies, which provided a new perspective for the study of English translation of Chinese classics.

Yanyan and Zhang Bin [9] selected two translations of $\mathrm{Wu}$ father and son and Li Zhaoguo to explain in detail the untranslatability of the language and cultural aspects of Emperor Internal Chinese Medicine, and proposed corresponding compensation methods such as transliteration, annotation and free translation, hoping to apply them to the teaching of TCM translation. The doctoral dissertation by $\mathrm{Li}$ Zhiyi [10] of Guangzhou University of Traditional Chinese Medicine is probably the first and only one on the translation of Emperor Internal Chinese Medicine so far. She also chose two full translations of $\mathrm{Wu}$ father and son and $\mathrm{Li}$ Zhaoguo to make a comparative study, but she only compared the translation of pricking blood therapy in the two translations from nine aspects: the understanding of collaterals, the principle of pricking blood, the tool of pricking blood, the method of pricking blood, the location of pricking blood, the amount and degree of bleeding, the reaction after pricking blood, the symptoms of pricking blood treatment and the taboo of pricking blood. This study holds that $\mathrm{Wu}$ father and son have relatively accurate and proper understanding of the original text because of their rich clinical experience. The language of the translation is more genuine and flexible, and they are good at using imperative sentences and active voice, but their shortcomings lie in missed translation and translation errors. Li Zhaoguo's translation adopts transliteration plus free translation, and the passive voice is often used in sentence pattern, which is more in line with the thinking habits of Chinese people. And his shortcoming lies in the lack of clinical experience. Jiang Jibiao and Zhang Bin [11], guided by the theory of functional translation, made a 
detailed comparison of Li Zhaoguo's and Veith's translations from the perspectives of translation strategies, coherence and loyalty, believing that Li Zhaoguo's translations are as old as the ancients in terms of rendering strategies, without decoration, strictly following the original text and adding words at will, while Veith's translations only pay attention to the main idea of the Inner Classic from the perspective of medical history and ignore a deep study of the meaning of individual words; in terms of translation methods, the basic concepts of Li Zhaoguo's translation are mainly transliteration, accompanied by explanations. In textual translation, literal translation is chiefly used supplemented by free translation, while Veith's translation adopts such methods as footnotes and sandwiches, which are beneficial for future generations to further consult; as for the loyalty of the translated text, $\mathrm{Li}$ Zhaoguo's version retains the characteristics of the original due to its high reliability and referring to various annotations, while Veith's version lacks the faithful reproduction of the original owing to the constraints of the time. In terms of coherence, Li Zhaoguo's translation is slightly weaker on fluency and lexical cohesion because of sticking to the expressive structure of the original text, while Veith's translation is graceful and coherent, which conforms to the reading habits of the target readers. Ye Xiao and Dong Minhua [12] created bilingual parallel corpus and used Paraconc software to extract relevant data. They selected English translation corpus of common pulse phenomena such as floating pulse, scattered pulse, deep pulse, deep-sited pulse, retarded pulse, infrequent pulse, rapid pulse, swift pulse, bounding pulse, thready pulse, long pulse, short pulse and feeble pulse in $\mathrm{Li}$ Zhaoguo's translation and Wen Shude's translation to extract, compare and sum up. The analysis shows that the two versions have their own advantages and disadvantages in the overall and detailed English translation of the pulse.

Under the guidance of schema theory and mapping theory, Liang $\mathrm{Hu}$ [13] uses relevant corpus analysis software to analyze the translation of metaphors, metonymies and synaesthetic figures of speech in General Questions by $\mathrm{Wu}$ father and son, Maoshing Ni and Li Zhaoguo, and to find out the translation differences among the three versions. The study finds that $\mathrm{Wu}$ father and son translation has a profound understanding of the original text and a strong explanatory power, but the translation is slightly lengthy; Maoshing Ni's translation is accessible and accurate, but the free translation is excessive; and Li Zhaoguo's translation has a variety of structures, which better retains the original style, but the interpretation is too simple. Li Jie [14] also selected three translations of $\mathrm{Wu}$ Father and Son, Maoshing $\mathrm{Ni}$ and $\mathrm{Li}$ Zhaoguo to make a comparative study. This study, by means of a questionnaire survey, allowed foreign students with TCM-based clinical practice to score their comprehension and acceptance satisfaction of the three translations of 10 sentences, and then calculate the total score. The results showed that Li Zhaoguo's translation scored the highest. The researchers further pointed out that translators should take into account the change of readers' horizons of expectation in the process of translation, thus changing translation strategies and methods accordingly to cater to readers' reading expectations. Ming ming [15] exploreed in detail the differences in academic background, bilingual competence, bilingual competence, bilingual competence, translation purposes and strategies of different translators from the perspective of translator subjectivity, thus giving the best annotations to the different features of the three versions of Veith, Wu father and son, and Li Zhaoguo.

\section{PAYING ATtENTION TO LANGUAGE TRANSFER SKILLS IN THE TRANSLATION OF EMPEROR INTERNAL CHINESE MEDICINE FROM THE MICRO-LEVEL}

Most of the studies on the translation and introduction of Emperor Internal Chinese Medicine concentrate on the microscopic translation methodology level, placing emphasis on the interlingual transfer skills of disease names, numerals, function words, figures of speech, and culture-loaded words in the original text. Some researchers, such as Li Yingzhao and Shi Yunzhong et al [16], investigated the translation of the names of some diseases in Emperor Internal Chinese Medicine. Liu Xianpeng and Yao Xin [17] respectively examined the English translation of semantic vague numerals and vague language. Some researchers, such as Huang Yu et al [18], paid attention to the translation of figures of speech of antithesis, ellipsis, anadiplosis and metaphor. Zhang Xuan and Shi Yunzhong [19] compared and analyzed the translation of the important culture-loaded words of traditional Chinese medicine in the first piece, The First Discussion of Ancient Nature, of Emperor Internal Chinese Medicine Suwen translated by $\mathrm{Li}$ Zhaoguo and Weiss. According to the statistics, 101 cultural-loaded words are found in this study, which were divided into three categories: culture-loaded words of noun type, culture-loaded words of verb type and culture-loaded words of adjective type. The differences between the two versions are also compared. Zhang Xuan [20] further expanded the research in master's thesis. She divided the culture-loaded words in Neijing into four categories, namely, culture-loaded words of people' name, culture-loaded words of traditional Chinese medicine terms, culture-loaded words of philosophical concepts related to traditional Chinese medicine, and other descriptive culture-loaded words, conducted a comparative empirical study on the English translation of culture-loaded words in two versions, and found that there were seven methods to translate these words in the two versions, including free translation, transliteration, literal translation, zero translation, translation variation, additional translation and subtraction.

\section{GUIDING AND ANALYZING THE TRANSLATION OF EMPEROR INTERNAL CHINESE MEDICINE BY USING ARIOUS CONTEMPORARY TRANSLATION THEORIES}

Numerous researchers have used various contemporary linguistic and translatological theories to explain, guide or analyze the translation of Emperor Internal Chinese Medicine, which marks the tentative combination of traditional Chinese medicine translation practice and contemporary translation theory and lays a good foundation for practitioners of traditional Chinese medicine translation to move forward rational translation. Each theory provides a new perspective for future researchers. These theories include skopostheorie, translation as adaptation and selection, 
translator subjectivity theory, deconstructive translation theory, functional equivalence theory, conceptual integration theory, adaptation theory, memetics, schema-mapping theory, reception theory, relevant adaptation theory, cultural manipulation theory, translation aesthetics, hermeneutics, eco-translatology, semiology, etc. For example, Wu Yina [21] et al studied the English version of Emperor Internal Chinese Medicine from the perspective of skopostheorie. Wu Chunyu, Wang Yinquan [22] et al respectively analyzed the English translation of main and collateral channels, acupuncture and moxibustion terms culture-loaded words in the two English versions of Emperor Internal Chinese Medicine Lingshu from the perspective of eco-translatology. Yuan Mi, Yao Xin et al [23] respectively analyzed the English translation of disease terms and Figure of Speech Enallage by using translation as adaptation and selection. More scholars used other different theories to study the translation of Emperor Internal Chinese Medicine from other dimension.

\section{CONCLUSION}

To sum up, we have achieved a great deal of gratifying results on the research of the translation and introduction of Emperor Internal Chinese Medicine. Moreover, it should be noted that the existing studies pay much attention to the micro and mesoscopic levels, or focus on the interlingual transfer skills at the linguistic level, or unilaterally pursue the integration of contemporary translation theory and traditional Chinese medicine translation. Moreover, these studies do not study further or deeper, therefore, the practicalness is strong, but the theoretical construction is insufficient. Existing studies have placed too much stress on the text itself and have been divorced from the social and cultural context of translation, introduction and transmission of Emperor Internal Chinese Medicine at that time and at present. As a result, the translation evaluation criteria are static and constant, while the influence of translator's subjective initiative, international political situation, sponsors and other hypertext factors on the selection and dissemination of translation strategies are ignored. As the quintessence of Chinese traditional culture, the translation, introduction and dissemination of traditional Chinese medicine classics represented by Emperor Internal Chinese Medicine are related to the grand strategy of Chinese cultural soft power construction. In the context of reality, traditional Chinese medicine is confronted with textual misinterpretation, cultural aphasia and ideological hegemony internationally. Therefore, it has great sample significance to study how to translate and disseminate the classics of traditional Chinese medicine represented by Emperor Internal Chinese Medicine by taking advantage of the international "traditional Chinese medicine fever" brought by Tu Youyou's Nobel Prize.

\section{REFERENCES}

[1] Liu Na, Wang Na, Zhang Tingting, Wang Zhimei. An Overview of the English Translation of Emperor Internal Chinese Medicine [J]. Asia-Pacific Traditional Medicine, 2014 (11): 2-3. (in Chinese)

[2] Xie Shuting. English Translation of Emperor Internal Chinese Medicine and Its Research Overview [J]. Journal of Yunnan
University of Traditional Chinese Medicine, 2012 (5): 67-70. (in Chinese)

[3] Zhang Lin, Wen Juan, Jiang Jichang. Study on Title Translation of Emperor Internal Chinese Medicine [J]. Asia-Pacific Traditional Medicine, 2015 (12): 3-4. (in Chinese)

[4] Shen Feiyu. Preliminary Study on the History of English Translation of Emperor Internal Chinese Medicine Suwen [J]. Journal of Hunan University of Technology (Social Science Edition), 2015 (2): 117-120. (in Chinese)

[5] Shi Yunzhong, Ma Jiming, Xu Zheng. The First English Translation of Huang Di Nei Jing [J]. Shanghai Journal of Translators for Science and Technology, 2002 (2): 46-49. (in Chinese)

[6] Li Zhaoguo. On C-E Translation of "The Yellow Emperor's Canon of Medicine" [J]. Chinese Science \& Technology Translators Journal, 2009 (4): 3-7. (in Chinese)

[7] Wang Xinyue. Enlightenment from English Translation of Traditional Chinese Medicine in Academic Exchange: Appreciation of $\mathrm{Li}$ Zhaoguo's English Translation of Emperor Internal Chinese Medicine [J]. Chinese Science \& Technology Translators Journal, 2012 (4): 36-39. (in Chinese)

[8] Huang Yu. A Study on the English Translation of Li Zhaoguo's Emperor Internal Chinese Medicine Suwen [D]. Northwest A\&F University, 2014. (in Chinese)

[9] Yan Yan, Zhang Bin. On the Untranslatability of Translation and Its Compensation Method Teaching from the Two Translations of Emperor Internal Chinese Medicine [J]. Lishizhen Medicine and Materia Medica Research, 2012 (5): 1267-1269. (in Chinese)

[10] Li Zhiyi. A Comparison of English Translation of Pricking Blood Therapy in Two Versions of Emperor Internal Chinese Medicine [D]. Guangzhou University of Chinese Medicine, 2013. (in Chinese)

[11] Jiang Jibiao, Zhang Bin. A Comparative Study of the English Versions of Emperor Internal Chinese Medicine Based on Functional Translation Theory $[\mathrm{J}]$. Chinese Journal of Basic Medicine in Traditional Chinese Medicine, 2014 (11): 1569-1572. (in Chinese)

[12] Ye Xiao, Dong Minhua. Discussion on the English Translation of the Name of "Pulse Condition" in Traditional Chinese Medicine Based on the Comparison of the English Translation of Pulse Condition in the two English Versions of Emperor Internal Chinese Medicine [J]. Chinese Journal of Basic Medicine in Traditional Chinese Medicine, 2015 (01): 94-96. (in Chinese)

[13] Liang Hu. A Contrastive Translation Study of Three Figures of Speech in the Three Versions of Emperor Internal Chinese Medicine Based on Schema-Mapping Theory [D]. Nanjing University of Chinese Medicine, 2012. (in Chinese)

[14] Li Jie. Questionnaire Analysis of Three English Versions of Emperor Internal Chinese Medicine from the Perspective of Reception Theory [J]. Guiding Journal of Traditional Chinese Medicine, 2015 (7): 106109. (in Chinese)

[15] Ming Ming. A Comparison of the Translator's Subjectivity in the Three Versions of Emperor Internal Chinese Medicine Suwen [J]. Journal of Kaifeng Institute of Education, 2014 (12): 254-256. (in Chinese)

[16] Li Yingzhao, Shi Yunzhong. Translation of Disease Names Related to "Bi" in Yellow Emperor's Canon of Medicine: An Interpretation in the Perspective of Approach of Translation as Adaptation and Selection [J]. China Science and Technology Information, 2009 (1): 221-223. (in Chinese)

[17] Liu Xianpeng, Yao Xin. A Study on the English Translation of Vague Language in Emperor Internal Chinese Medicine from the Perspective of Relevance Adaptation Theory [J]. Chinese Journal of Basic Medicine in Traditional Chinese Medicine, 2015 (06): 747-749. (in Chinese)

[18] Huang Yu. Review of English Translation Study on Figure of Speech in Emperor Internal Chinese Medicine [J]. Success, 2013 (23): 304. (in Chinese)

[19] Zhang Xuan, Shi Yunzhong. English Translation of Culture-specific Lexicon Commonly Seen in Emperor Internal Chinese Medicine [J]. Chinese Journal of Integrated Traditional and West, 2008 (10): 941944. (in Chinese) 
[20] Zhang Xuan. A Study on the English Translation of Culture-loaded Words in Emperor Internal Chinese Medicine [D]. Nanjing University Of Chinese Medicine, 2009. (in Chinese)

[21] Wu Yina. A Comparison of Two English Translations of Emperor Internal Chinese Medicine from Skopos Theory [D]. Fudan University, 2012. (in Chinese)

[22] Wu Chunyu, Zhang Bin. Research on Strategies of Several English Translations of Emperor Internal Chinese Medicine from the Perspective of Eco-translatology [J]. Guiding Journal of Traditional Chinese Medicine, 2014(13): 104-106. (in Chinese)

[23] Yuan Mi, Yao Xin. On the English Translation of Figure of Speech Enallage in Neijing From the Perspective of Translation as Adaptation and Selection [J]. Lishizhen Medicine and Materia Medica Research, 2014 (05): 1222-1224. (in Chinese) 